\title{
CHALLENGES IN MAGNETO-OPTIC MEDIA FOR ULTRA HIGH DENSITY RECORDING (Invited)
}

\author{
C.-J. LIN \\ IBM Research Division, Almaden Research Center, San Jose, CA 95120-6099, USA
}

\section{Introduction}

One of the key thrusts for an ever improving data storage technology is to reduce the cost per megabyte of data, with no exception in magneto-optical (MO) storage. The reduction in cost/Mbyte is accomplished primarily by increasing the areal data bit density on the storage medium. In order to achieve ultra high density recording in future magneto-optical storage there requires technology improvements in many areas: such as laser, media, and servo performance. The key challenges in media, for the ultra high density MO storage device to be capable of retrieving the stored data with a final bit error rate of $10^{-12}$ or less, are primarily in the disk substrate and the magneto-optical recording layer.

\section{Substrate}

In order for the servo system to be able to position the submicron read/write laser light spot very accurately on the MO disk, the MO disk substrate has to provide relief structures, in the forms of either continuous lands and grooves or discrete pits with feature dimension comparable to the laser wavelength used in the drive. With much shorter wavelength like that of a blue laser light to be used in ultra high density recording, the ability to form such servo tracking structures of sufficiently good quality is quite a challenge, especially for forming such structures directly on glass substrates. Moreover, in order to ensure sufficiently low hard errors that result from defects in the MO coating, the substrate surface of data-recorded regions has to be flat and free from defects.

\section{Magneto-Optical recording Material}

There are two key requirements for a candidate MO recording material to be used in the future ultra high density recording. One is to have large Kerr or Faraday rotations at short wavelengths. The other is to have sufficiently large perpendicular magnetic anisotropy and coercivity. Furthermore, in order to have a large readback signal amplitude and a sufficiently low level of shot noise, there will be a necessity in reducing the thermal sensitivity of the disk media to allow readback at high laser power. Ultra high density recording also requires improved magnetic properties and a better media uniformity, together with improvements in thermomagnetic writing techniques, to ensure a smaller transition jitter. 
The candidate MO recording materials for future ultra high density recording are very likely limited to the following three choices: amorphous rare-earth transition-metal (RE-TM) alloys, polycrystalline garnet, and polycrystalline Co/Pt multilayers. For each candidate material the following issues will be discussed: magnetic properties such as perpendicular anisotropy, nucleation and domain wall motion coercivities, saturation magnetization, and Curie temperature; magneto-optical properties such as Kerr or Faraday rotations at shorter wavelengths; structure properties such as amorphism, crystallinity, crystalline orientation and the thermal stability of these structures; and chemical properties such as oxidation and corrosion resistance. The influence of these properties on the minimum domain size, written transition jitter, write sensitivity, readback signal amplitude, write/erase cyclability, environmental stability, and manufacturability will be addressed. 